\title{
Decision Analysis of E-commerce Closed-loop Supply Chain with Different Recycling Modes
}

\author{
Sijia Liu ${ }^{1, a}$, Yanting Huang*2,b \\ ${ }^{1}$ College of Management, Shenzhen University, Shenzhen, China \\ ${ }^{2}$ College of Management, Shenzhen University, Shenzhen, China
}

\begin{abstract}
According to an e-commerce closed-loop supply chain dominated by manufacturers, which is composed of manufacturers and e-commerce platforms, divided into three different recovery mode: manufacturers recycling mode alone, electric business platform recycling mode alone, and manufacturers and electric business platform mixed mode, using the game theory to solve, compares three closed-loop supply chains found: (1)When the recycling price sensitivity is high, the optimal strategy of the manufacturer is the manufacturers recycling mode alone; when the price sensitivity of recycling is low, the manufacturer's best strategy is the independent recycling mode of the e-commerce platform. No matter how sensitive the recycling price is, the profits of the manufacturer under the mixed recycling mode are always lower than those under the other two recycling modes. (2) When the recycling price sensitivity is high, the optimal strategy of the electric business platform is the mixed recycling mode; when the price sensitivity of recycling is low, the optimal solution of the electric business platform is the independent recycling mode of the electric business platform. (3) When the recovery price sensitivity is low, the best strategy of both is the separate recycling mode of the electric business platform.
\end{abstract}

\section{Introduction}

In recent years, people's awareness of environmental protection has been increasing and sustainable development has been deeply implemented. Recycling waste products has received wide attention from society, and more and more enterprises have joined the pace of recycling waste products. Recycling waste products from customers and processing or remanufacturing them through e-commerce platforms can improve transaction efficiency, reduce recycling costs and optimize resource allocation. The e-commerce platform and the closed-loop supply chain system are combined to form the ecommerce closed-loop supply chain(EC-CLSC). In this system, the e-commerce platform replaces the third-party recyclers and retailers, recycling the waste products and selling the secondary products. The platform directly transfers the recycled waste products to the manufacturer for decomposition, disassembly, assembly, and remanufacturing, and the secondary products are sold through the e-commerce platform. At present, there are many domestic recycling platforms, such as AHS DEVICE, Le recycling, Wo recycling.

In real economic life, the ways of recycling waste products are complex and diverse. Therefore, this paper studies different recycling modes: manufacturer alone recycling mode, e-commerce platform alone recycling mode, and manufacturer and e-commerce platform mixed recycling mode, and discusses the pricing decision and performance of manufacturer and e-commerce platform under three different modes.

The research on an e-commerce platform, EC-CLSC, and recycling mode in academic circles has achieved fruitful results. It can be elaborated mainly from the following aspects :(1) Research on an e-commerce platform. Gopalakrishnan et al. [1] defined the optimal waste flow by proposing a blockchain platform and optimization model to improve the profit of suppliers. Aspa et al. [2] studied business models such as C2B and B2B2C to allow customers to participate in the company's supply chain and business operations. Adrian et al. [3] investigated consumers' acceptance of three circular economy practices in East and Southeast Asia: using shared platforms, purchasing recycled goods, and buying secondary products. Dutta et al. [4] studied returns in the Indian e-commerce market by proposing a multi-objective logistics network model. (2) E-commerce supply chain decisions. From the perspective of capital constraint electric business platforms operation, Wang [5] studied the impact of different capital constraint lifting methods on ecommerce supply chain pricing and service decisions. Xiao [6], on the other hand, focused on a supply chain composed of an e-commerce platform and multiple retailers and studied how e-commerce platforms use digital authorization to promote retailers' sales efforts. (3) Decision of EC-CLSC. Wang et al. [7] designed two experiments to empirically study the influence mechanism of green information on the willingness to participate in recycling websites. Wang et al. [8] studied the

${ }^{*}$ b Corresponding author: ythuang4028@szu.edu.cn 
coordination mechanism of low-carbon e-commerce closed-loop supply chain, Gong et al. [9] researched the impact of deposit refund system on the optimal decision and performance of a single network platform and a single manufacturer in an EC-CLSC. Suvrat [10] studied the true environmental benefits of an online platform in a CLSC in the context of an Internet-driven $\mathrm{C} 2 \mathrm{C}$. Jia and $\mathrm{Li}$ [11] looked at the choice of platform distribution channel model and analyzed a CLSC consisting of an e-retailer's online marketplace (i.e., platform services) and selfowned stores. Xiang and $\mathrm{Xu}$ [12] studied the CLSC dynamic involving Internet service platforms, mainly discussing how supply chain members choose the optimal cooperation model to achieve Pareto improvement. (4) Compare the recycling modes of different channels. Literature [13-17] is based on the manufacturer-led singlechannel recycling mode. Savaskan et al. [13-14] studied the selection of manufacturer's recycling channels under three recycling modes: direct recycling by the manufacturer, recycling by the manufacturer entrusted to the seller, and recycling by a third party. Huang et al. [15] studied the optimal strategy of CLSC with dual recycling channels composed of vendors and third parties. Liu et al. [16] established three dual recycling modes and concluded through analysis that the dual recycling mode of manufacturer and seller was the best. Esmaeili et al. [17] studied the recycling strategies of the two-level closedloop supply chain in the short and long term for manufacturer recycling and seller recycling. Hong et al. [18] established three models in the CLSC in which manufacturers, retailers, and third-party recyclers are respectively responsible for recycling, and studied the coordination strategy of the system. Zhang [19] considers the decision-making of supply chain members in decentralized and centralized situations and aims to integrate the return problems caused by the product quality and quality problems into the design of the dualchannel coordination mechanism of the CLSC.

The above studies are of electricity made outstanding contributions to the further development of the CLSC, but it is insufficient, mainly reflects in: most studies only consider EC-CLSC itself, not to other factors into the CLSC, did not consider other factors and electric business platform at the same time discusses the factors impact on the electricity business decisions and performance of CLSC. Therefore, this paper, based on the manufacturer as the leading, considers the different recycling modes of the EC-CLSC, including the manufacturer alone recycling, the e-commerce platform alone recycling, and the CLSC model of both recycling at the same time, and compares and analyzes different recycling modes, to obtain some useful conclusions.

\section{Model Building}

Suppose an EC-CLSC consists of a manufacturer and an e-commerce platform. The recycling methods of waste products can be divided into three categories: manufacturers directly recycle at the recycling price of $P_{m}^{M} \quad$ (M recycling mode), manufacturers entrust ecommerce platforms to recycle at the recycling price of $P_{e}^{E} \quad$ (E recycling mode), and manufacturers and ecommerce platforms respectively recycle at the recycling price of $P_{m}^{M E}$ and $P_{e}^{M E}$ and simultaneously recycle (ME mixed recycling mode), all of which are manufacturer-led. Recycled waste products through the above three channels are finally handed over to manufacturers for processing and remanufacturing, and remanufactured products are sold through e-commerce platforms. Manufacturers only remanufacture recycled products, not produce new ones.

The main parameters of this paper are set as follows:

$P_{m}$ : Unit recovery price of waste products recycled by the manufacturer;

$P_{e}$ : Unit recycling price of waste products recycled on e-commerce platform;

$C_{r}$ : Manufacturer's unit cost of remanufacturing using recycled products;

$P$ : The unit selling price of remanufactured products;

$\beta$ : Sensitivity of consumers to product recycling price;

$\rho$ : Unit commission given by the manufacturer to the e-commerce platform for recovery;

$\pi_{m}$ and $\pi_{e}$ : represent the profits of the manufacturer and the e-commerce platform respectively, and the superscript M, E, and ME represent the three recycling modes respectively.

\subsection{Manufacturer alone recovery mode}

When the manufacturer recycles the waste products separately (as shown in Figure 1), the manufacturer decides the recycling price of the waste products $-P_{m}^{M}$. In this case, the function of recycling quantity is: $q^{M}=$ $\alpha+\beta P_{m}^{M} \cdot \alpha(\alpha \geq 0)$ represents the minimum amount of recycling in the market, generally refers to the number of waste products that consumers voluntarily hand in free of charge in environmental awareness, and $\beta(\beta>0)$ represents the coefficient of the impact of recycling price on recycling quantity. In this mode, the manufacturer's profit function is

$$
\pi_{m}^{M}=\left(P-C_{r}-P_{m}^{M}\right)\left(\alpha+\beta P_{m}^{M}\right)
$$

Easy to know $\pi_{m}^{M}$ is a concave function of $P_{m}^{M}$, which has a maximum. Get

$$
P_{m}^{M}=\frac{\left(P-C_{r}\right) \beta-\alpha}{2 \beta}
$$

Substitute this expression into (1) to get $\pi_{m}^{M}=$ $\frac{\left[\left(P-C_{r}\right) \beta+\alpha\right]^{2}}{4 \beta}$. 


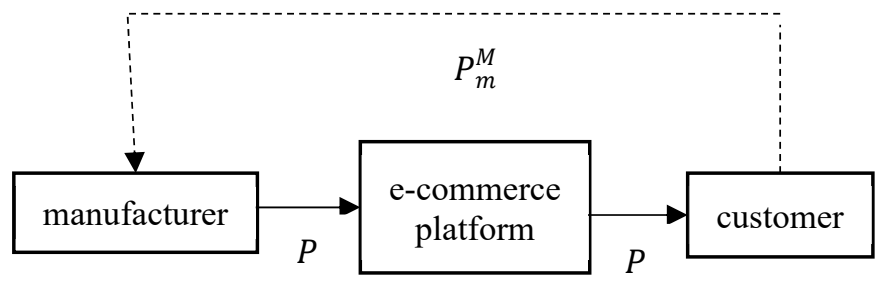

Fig 1. Manufacturer alone recovery mode1

\subsection{Separate recycling mode on e-commerce platform}

In the separate recycling mode of the e-commerce platform (as shown in Figure 2), the manufacturer entrusts the e-commerce platform to recycle the used products at the recycling price of $P_{e}^{E}$, and the manufacturer gives the e-commerce platform a commission for each unit of recycled products, denoted by $\rho^{E}$. The recycled quantity of the e-commerce platform is $q^{E}=\alpha+\beta P_{e}^{E}$, and the operating cost of the e-commerce platform is $C^{E}=\frac{k \rho^{E^{2}}}{2}$. In this recycling mode, the manufacturer decides the recycling price $--P_{e}^{E}$, and the e-commerce platform decides the commission $\rho^{E}$, according to $P_{e}^{E}$.

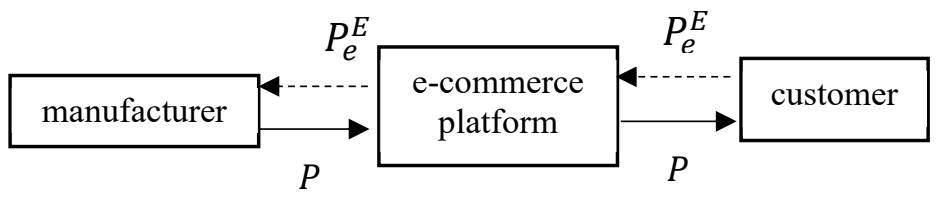

Fig 2. Individual recycling mode of e-commerce platform

At this point, the profit function of the manufacturer and the e-commerce platform are respectively

$$
\begin{gathered}
\pi_{m}^{E}=\left(P-C_{r}-\rho^{E}-P_{e}^{E}\right)\left(\alpha+\beta P_{e}^{E}\right) \\
\pi_{e}^{E}=\rho^{E}\left(\alpha+\beta P_{e}^{E}\right)-\frac{k \rho^{E^{2}}}{2}
\end{gathered}
$$

According to the backward induction, the optimal decision is

$$
\begin{gathered}
P_{e}^{E}=\frac{k P \beta-k \beta C_{r}-2 \alpha \beta-k \alpha}{2 \beta^{2}+2 k \beta} \\
\rho^{E}=\frac{P \beta+\alpha-\beta C_{r}}{2 \beta+2 k} \\
\pi_{m}^{E}=\frac{k\left(\alpha+P \beta-\beta C_{r}\right)^{2}}{4 \beta(\beta+k)} \\
\pi_{e}^{E}=\frac{k\left(\alpha+P \beta-\beta C_{r}\right)^{2}}{8(\beta+k)^{2}}
\end{gathered}
$$

\subsection{Mixed recycling mode of manufacturers and e-commerce platforms}

When the manufacturer and electric business platform recycle at the same time (as shown in figure 3) with recycling price respectively $P_{m}^{M E}$ and $P_{e}^{M E}$. Due to the competition between different recycling channels, the manufacturer's recovery quantity function is: $q_{m}^{M E}=$ $\beta P_{m}^{M E}-s P_{e}^{M E}$, the one about the electric business platform is: $q_{e}^{M E}=\beta P_{e}^{M E}-s P_{m}^{M E} . s$ represents to the influence degree of the recycling competition between parties, the greater $s$ said they're own recycled by the greater the competitive impact of the electric business platform for recycling. Therefore, to make sense of this calculation, let's say, $\beta>s>0$.

Now, the manufacturer's profit function is as follows:

$$
\begin{array}{r}
\pi_{m}^{M E}=\left(P-C_{r}-P_{m}^{M E}\right)\left(\beta P_{m}^{M E}-s P_{e}\right)+\left(P-C_{r}-\rho^{M E}-\right. \\
\left.P_{e}^{M E}\right)\left(\beta P_{e}^{M E}-s P_{m}^{M E}\right)
\end{array}
$$

Profit function of e-commerce platform is:

$$
\pi_{e}^{M E}=\rho^{M E}\left(\beta P_{e}^{M E}-s P_{m}^{M E}\right)-\frac{k \rho^{M E^{2}}}{2}
$$

The optimal decision is obtained by backward induction

$$
\begin{gathered}
P_{m}^{M E}=\frac{P-C_{r}}{2} \\
P_{e}^{M E}=\frac{P-C_{r}}{2} \\
\rho^{M E}=\frac{\left(P-C_{r}\right)(\beta-s)}{2 k} \\
\pi_{m}^{M E}=\frac{\left(P-C_{r}\right)^{2}(\beta-s)[2 k-(\beta-s)]}{4 k} \\
\pi_{e}^{M E}=\frac{\left(P-C_{r}\right)^{2}(\beta-s)^{2}}{8 k}
\end{gathered}
$$

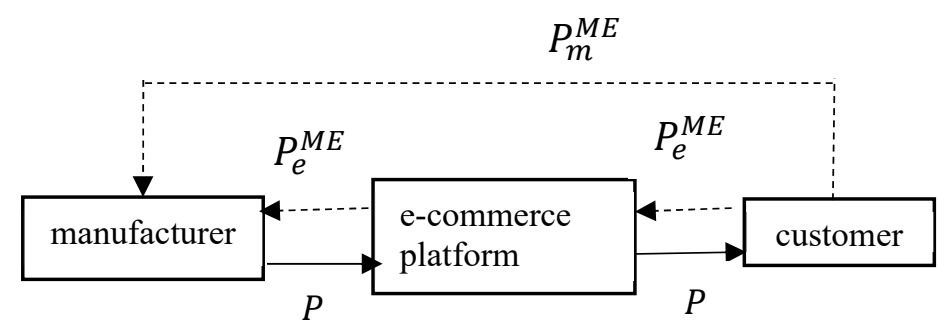

Fig 3. Mixed recycling mode of manufacturer and e-commerce platform 2 


\section{Different Recycling Mode Comparative Analysis}

The following paragraphs will compare the recovery price and commission under the three recovery modes and discuss the advantages and disadvantages of the three modes.

Conclusion 1. The recycling price in the mixed recycling mode of manufacturer and e-commerce platform is higher than that in the single recycling mode of the manufacturer and the single recycling mode of ecommerce platform, i.e. $P_{m}^{M}<P_{m}^{M E}, P_{e}^{E}<P_{e}^{M E}$.

Proof: $P_{m}^{M}=\frac{\left(P-C_{r}\right) \beta-\alpha}{2 \beta}=\frac{P-C_{r}}{2}-\frac{\alpha}{2 \beta}<\frac{P-C_{r}}{2}=P_{m}^{M E}, P_{e}^{E}-$ $P_{e}^{M E}=-\frac{\beta\left(P-C_{r}\right)(2 \beta+k)+2 \alpha \beta+k \alpha}{2 \beta(\beta+k)}<0$.

Conclusion 2. In the latter two recovery modes, manufacturers give unit commission to the e-commerce platform, and the selection strategy of unit commission is as follows:

(1) when $0<\beta<\frac{\left.s+\sqrt{s^{2}+4\left[s k+k \alpha /\left(P-C_{r}\right)\right.}\right]}{2}, \rho^{E}>\rho^{M E}$. That is, the unit commission under E recovery mode is higher than that under ME mixed recovery mode.

(2) when $\beta>\frac{\left.s+\sqrt{s^{2}+4\left[s k+k \alpha /\left(P-C_{r}\right)\right.}\right]}{2}, \rho^{E}<\rho^{M E}$. That is, the unit commission under $\mathrm{E}$ recovery mode is lower than that under ME mixed recovery mode.

Proof: $\rho^{E}-\rho^{M E}=\frac{\left(P-C_{r}\right)\left(-\beta^{2}+\beta s+s k\right)+k \alpha}{2 k(\beta+k)}$. According to the calculation, when $0<\beta<$ $\frac{\left.s+\sqrt{s^{2}+4\left[s k+k \alpha /\left(P-C_{r}\right)\right.}\right]}{2}, \rho^{E}>\rho^{M E} ;$ when $\beta>$ $\frac{\left.s+\sqrt{s^{2}+4\left[s k+k \alpha /\left(P-C_{r}\right)\right.}\right]}{2}, \rho^{E}<\rho^{M E}$. This indicates that, for the manufacturer, when $0<\beta<\frac{\left.s+\sqrt{s^{2}+4\left[s k+k \alpha /\left(P-C_{r}\right)\right.}\right]}{2}$, the unit commission was given by the manufacturer to the e-commerce platform is higher than the unit commission given by the manufacturer to the e-commerce platform in the separate recycling mode than in the mixed recycling mode; when $\beta>\frac{\left.s+\sqrt{s^{2}+4\left[s k+k \alpha /\left(P-C_{r}\right)\right.}\right]}{2}$, the unit commission was given by the manufacturer to the ecommerce platform in the separate recycling mode is lower than that in the mixed recycling mode. From the perspective of the e-commerce platform, it is the opposite.

\section{Numerical Analysis}

To further describe the above conclusions, this section carries out detailed verification through numerical analysis.

Assuming $P=3, C_{r}=1, \alpha=0.4, k=0.8$, and $s=0.3$ is subsumed into the solution of recycling price, commission, and profit balance of manufacturers and ecommerce platforms under different recycling modes. The accuracy of the conclusion can be vividly verified by using
Excel to draw a graph and taking the sensitivity of recycling price $-\beta$ as a variable.

By substituting the value into the manufacturer's recovery price, we can get $P_{m}^{M}=\frac{\beta-0.2}{\beta}$ and $P_{m}^{M E}=1$ under the three recovery modes. Excel is used to draw the diagram as shown in Figure 4. It can be seen that the manufacturer's recovery price in the ME mixed recovery mode is always greater than that in the $\mathrm{M}$ recovery mode.

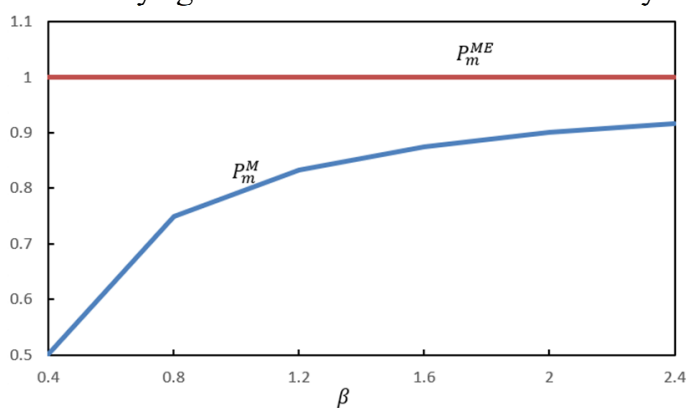

Fig 4. Relationship between manufacturer's recycling price under different recycling modes

At this time, the recycling price of the e-commerce platform is $P_{e}^{E}=\frac{0.4 \beta-0.16}{\beta^{2}+0.8 \beta}$ and $P_{e}^{M E}=1$. Excel is used to draw the diagram as shown in Figure 5. It is easy to know that the recycling price of the e-commerce platform under the ME mixed recycling mode is always higher than that under the E recycling mode. The comparison between Fig. 4 and Fig. 5 shows that the recycling price in the ME mixed recycling mode is higher than that in the $M$ recycling mode and the $\mathrm{E}$ recycling mode. In the $\mathrm{ME}$ mixed recycling mode, the recycling price of the manufacturer and the e-commerce platform is equal. This is because when manufacturers and e-commerce platforms collect at the same time, there is a competitive relationship that can keep prices at a level favorable to both sides.

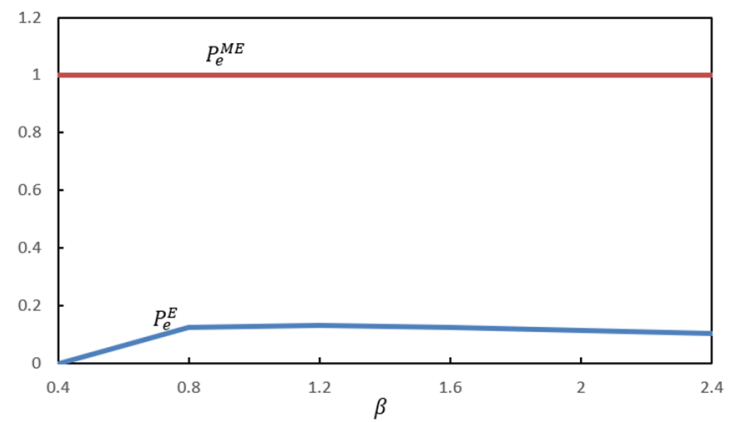

Fig 5. The relationship between recycling prices of ecommerce platforms under different recycling modes

Unit commission under $\mathrm{E}$ recovery mode and $\mathrm{ME}$ mixed recovery mode can be expressed as $\rho^{E}=\frac{\beta+0.2}{\beta+0.8}$ and $\rho^{M E}=\frac{\beta-0.3}{0.8}$. The Excel diagram is shown in Figure 6 . At that time of $0<\beta<1.2$, the unit commission under E recovery mode is higher than the unit commission under ME mixed recovery mode. At that time of $\beta>1.2$, the unit commission under the $\mathrm{E}$ recovery mode was much lower than the unit commission under the ME mixed recovery mode. 


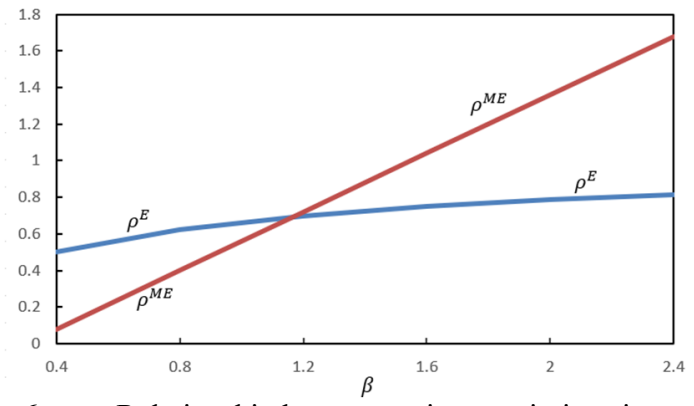

Fig 6. Relationship between unit commission size under different recovery modes

Substitute the value into the manufacturer's profit to obtain $\pi_{m}^{M}=\frac{(2 \beta+0.4)^{2}}{4 \beta}, \pi_{m}^{E}=\frac{0.2(0.4+2 \beta)^{2}}{\beta(\beta+0.8)}$ and $\pi_{m}^{M E}=$ $\frac{(\beta-0.3)(1.9-\beta)}{0.8}$. The Excel diagram is shown in Figure 7. The manufacturer's profit under the ME recovery mode is always lower than that under the $M$ recovery mode and the E recovery mode. At that time of $0<\beta<0.719$, the manufacturer's profit under $\mathrm{E}$ recovery mode is higher than that under $\mathrm{M}$ recovery mode; at the time of $\beta>$ 0.719 , the manufacturer's profit under the recycling mode $\mathrm{E}$ is much lower than that under the recycling mode $\mathrm{M}$. Therefore, for the manufacturer, when consumers are less sensitive to the recycling price, the manufacturer should choose $\mathrm{E}$ recycling mode, otherwise, the manufacturer should choose $\mathrm{M}$ recycling mode.

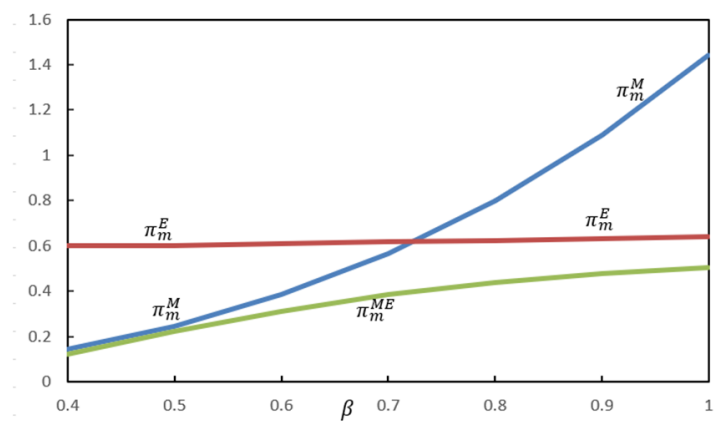

Fig 7. Relationship between manufacturers' profits under different recovery modes

At this point, the profit of the electric business platform can be expressed as $\pi_{e}^{E}=\frac{0.1(0.4+2 \beta)^{2}}{(\beta+0.8)^{2}}$ and $\pi_{e}^{M E}=\frac{(\beta-0.3)^{2}}{1.6}$. Excel is used to draw the diagram as shown in Figure 8 . When $0<\beta<0.702$, the profit of electric business platform under the $\mathrm{E}$ recycling mode is greater than that under the ME mixed recycling mode; when $\beta>0.702$, the profit of the electric business platform under the E recycling mode was far less than that under the ME mixed recycling mode. Therefore, for the ecommerce platform, when customers are less sensitive to the recycling price, the e-commerce platform should choose the $\mathrm{E}$ recycling mode, otherwise, it should choose ME mixed recycling mode.

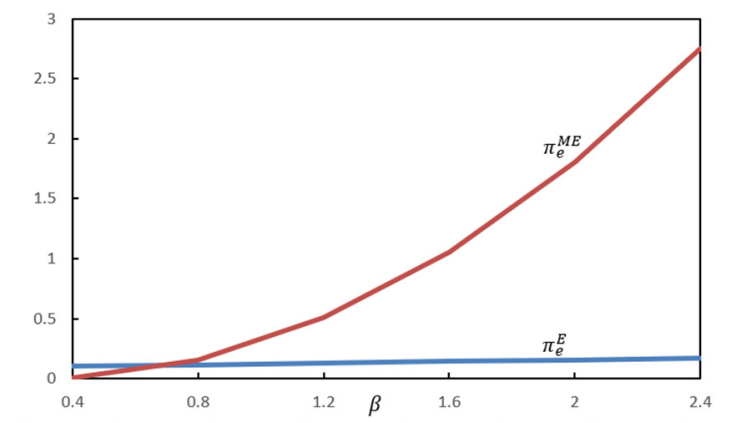

Fig 8. Relationship between profit sizes of e-commerce platforms under different recycling modes

\section{Conclusion}

To improve the utilization efficiency of waste products, the government encourages manufacturers to recycle and remanufacture. At the same time, with the development of e-commerce platforms, it has become an inevitable trend to incorporate e-commerce platforms into CLSC. In this background, this paper aimed at a consisting of manufacturers and electric business platform, dominated by manufacturers of EC-CLSC, through the study of three different recovery mode: manufacturers recycling mode alone, electric business platform recycling mode alone, and manufacturers and mixed electric business platform model. We found that no matter how price-sensitive, manufacturers and electric business platforms for the mixed mode of two recycling prices are higher than the other two kinds of recycling mode of the recycling price. But manufacturer's units pay commissions and both profits related to the recycling price sensitivity. At that time of $0<\beta<1.2$, the unit commission under the single recycling mode of the electric business platform is higher than the unit commission under the mixed recycling mode of the manufacturer and electric business platform. At that time of $\beta>1.2$, the unit commission under the separate recycling mode of the e-commerce platform was much lower than the unit commission under the mixed recycling mode of manufacturer and ecommerce platform. When $0<\beta<0.719$, the manufacturer's profit under the independent recovery mode of the e-commerce platform is higher than that under the independent recovery mode of the manufacturer. When $\beta>0.719$, the manufacturer's profit in the separate recovery mode of the electric business platform is far lower than that in the separate recovery mode. At the moment of $0<\beta<0.702$, the electric business platform's profit under the independent recycling mode is greater than that under the mixed recycling mode of the manufacturer and the electric business platform. At the moment of $\beta>0.702$, the electric business platform's profit under the separate recycling mode of the ecommerce platform was far less than that under the mixed recycling mode of the manufacturer and the electric business platform. For manufacturers and e-commerce platforms, the level of profit determines the choice of recycling mode. Therefore, the optimal choice for manufacturers and the electric business platform is the independent recycling mode of e-commerce platforms at that time of $0<\beta<0.702$; the two options differed at 
the time of $\beta>0.702$. Therefore, when $0<\beta<0.702$, to realize the effective recycling and reuse of waste products, the independent recycling mode of the electric business platform is the best choice of both.

Based on the analysis of different recycling channels of EC-CLSC, this paper doesn't discuss the advantages and disadvantages of different recycling channels under different power structures. In the next step, based on the research of this paper, it will be further expanded to analyze the enterprise decision-making problem of different ways of recycling channels under different power structures.

\section{Acknowledgment}

This research is supported by National Natural Science Foundation of China under Grant 72001147 and Guangdong Planning Project of Philosophy and Social Science of China under Grant GD19YGL18.

\section{References}

1. Gopalakrishnan, P.K., Hall, J., Behdad, S. (2021) Blockchain-based solid waste management system for comprehensive management. Waste Management., 120: 594-607.

2. Aspara, J., Grant, David, B., Holmlund, M. (2020) Consumer Involvement in Supply Networks: A Cubic Typology of C2B2C and C2B2B Business Models. Industrial Marketing Management., (prepublish).

3. Adrian, T.H., Kuah, Assoc, P., Wang, Assoc, Professor, P. (2020) Circular Economy and Consumer Acceptance: An exploratory study in East and Southeast Asia. Journal of Cleaner Production., 247: $1-13$.

4. Dutta, P., Mishra, A., Khandelwal, S., et al. (2020) A Multiobjective Optimization Model for Sustainable Reverse Logistics in Indian E-Commerce Market. Journal of Cleaner Production., 249: 1-13.

5. Wang, Y., Yu, Z., Jin, M. (2019) E-commerce supply chains under capital constraints. Electronic Commerce Research and Applications., 35: 1-14.

6. Xiao, D., Kuang, X., Chen, K. (2020) E-Commerce supply chain decisions under platform digital empowerment: induced demand. Computers \& Industrial Engineering., 150: 1-13.

7. Wang, C., Zhu, T., Yao, H. et al. (2020) The Impact of Green Participation Intention on Consumers in Online Recycling: An Experimental Study. Sustainability., 12: $1-16$.

8. Wang, Y., Fan, R., Shen, L., Altruistic preference: Recycling Decisions of Low-carbon E-Commerce Closed-Loop Supply Chain under Government subsidy mechanism. Journal of Cleaner Production., 259: 1-13.

9. Gong, Y., Chen, M., Wang, Z., et al. (2021) With or without deposit-refund system for a network platformer - LED electronic closed-loop supply chain. Journal of Cleaner Production., 281: 1-14.
10. Dhanorkar, S. (2019) Environmental Benefits of internet-enabled C2C Closed-loop Supply Chains: A Quasi-experimental Study of Craigslist. Journal of Management Science., 65: 660-680.

11. Jia, D., Li, S. (2020) Optimal decisions and distribution channel choice for closed-loop supply chain when electronic retailer offers online marketplace. Journal of Cleaner Production., 265: 113.

12. Xiang, Z., Xu, M. (2019) Dynamic cooperation strategies for the Closed-loop Supply Chain Platform involving the Internet Service Platform. Journal of Cleaner Production., 220:1180-1193.

13. Savaskan, R C., Bhattacharya, S., Wassenhove, L N V. (2004) Closed-loop supply chain models with product remanufacturing. Management Science., 50: 239-252.

14. Savaskan, R C., Wassenhove, L N. (2006) The case of competing retailers. Management Science., 52: 1-14.

15. Huang M., Song M., Lee L H., et al. (2013) Analysis of Closed-Loop Supply Chain with Dual Recycled Channel. International Journal of Production Economics., 144: 510-520.

16. Liu L, Wang Z, Hong X, et al. (2017) Collection effort and reverse channel choices in a closed-loop supply chain. Journal of Cleaner Production., 144: 492-500.

17. Esmaeili, M., Allameh, G., Tajvidi, T. (2016) Using Game Theory for Analysing Models in Closed-loop Supply Chain from Short-and Long-term Perspectives. International Journal of Production Research., 54: 1-18.

18. Hong, X., Xu, L., Du, P., et al. (2015) Joint Advertising, Pricing and Collection Decisions in a Closed-Loop Supply Chain. International Journal of Production Economics., 167: 12-22.

19. Zhang, Z., Liu, S., Niu, B. (2020) Coordination Mechanism for Product Quality Analysis with Closed-Loop Supply Chains. Journal of Cleaner Production., 248: 1-17. 